\title{
Different Core-Hole Lifetime and Screening in the Surface of W(110)
}

\author{
D. M. Riffe, G. K. Wertheim, and P. H. Citrin \\ AT\& T Bell Laboratories, Murray Hill, New Jersey 07974
}

(Received 14 June 1989)

\begin{abstract}
High-resolution $4 f$ photoemission spectra from clean W(110) show that the natural lifetime width and the (electron-hole)-pair singularity index are both larger in the first atomic layer than in the bulk. Phonon broadening for the surface and bulk components are smaller than theoretical estimates, and little excess broadening is detected in the surface layer. These findings are very different from the conventional picture of surface-atom core-level line shapes and have implications extending to other systems.
\end{abstract}

PACS numbers: 73.20.At, 79.60.Cn

It is now well established that core-level photoemission is a sensitive probe for studying the effects of the reduced coordination at the surface. ${ }^{1}$ With this spectroscopy the primary focus has been on the shift of the surface-atom core-level binding energy, whose sign and magnitude have been shown to be directly related to the narrowing and shape of the surface density of states. ${ }^{2-4}$ Studies of this shift as a function of material, crystal orientation, reconstruction, depth below the surface (i.e., subsurface layer shifts), adsorbate, and coverage exemplify the wide range of activity and interest in this area. ${ }^{1,5,6}$ However, the investigation of other manifestations of the reduced coordination at the surface, such as changes in core-hole lifetime, conduction-electron screening, and phonon broadening has been hampered by a lack of adequate experimental resolution and statistics. Indeed, while the excess width of the surface-atom core level in just a single metal, $\mathrm{W}$, has been tentatively ascribed to additional surface phonon broadening, crystal-field splitting, reconstruction, and subsurface contributions, ${ }^{5,7,8}$ no measurement has yet succeeded in clearly identifying the broadening mechanisms operative at the metal surface.

In this Letter we report on high-intensity, high-resolution core-level photoemission measurements from a clean W(110) surface. We find that the major contribution to the increased surface linewidth in this metal involves none of the previous suggestions, but is, instead, enhanced lifetime broadening resulting from the more localized $5 d$ electrons in the narrowed surface conduction band. In addition, we find differences in core-hole screening between the surface and bulk components which are manifest as a measurable difference in the shape of the $(e-h)$-pair excitation tail of the photopeak. These novel results are not specific to $W$, and should apply to a large number of systems in which conduction electrons dominate the core-hole decay.

The single-crystal sample of dimensions $4 \times 20 \times 0.2$ $\mathrm{mm}^{3}$ was cleaned by resistive heating to $1520 \mathrm{~K}$ in $10^{-7}$ Torr of oxygen followed by flashing to $2300 \mathrm{~K}$ in a vacuum of $1 \times 10^{-10}$ Torr. After cooling to either 210 or 310 $\mathrm{K}$, which took only seconds due to the large difference in thermal mass between the sample and its mount, photoemission data were collected using the AT\&T Bell La- boratories $6-\mathrm{m}$ toroidal-grating monochromator (TGM) on the vacuum-ultraviolet ring of the National Synchrotron Light Source. A $100-\mathrm{mm}$ voltage standing-wave hemispherical analyzer was used with a pass energy of 2 $\mathrm{V}$, which gives a resolution of $40 \mathrm{meV}$ (FWHM). The high intensity of the monochromatized radiation $(70 \mathrm{eV}$ for all measurements reported here) allowed single 2.5eV scans with high statistics to be obtained in only 4 min, during which time the average surface $\mathrm{H}$ contamination is estimated not to exceed 0.01 monolayer.

The total instrumental resolution was determined from thirteen independent measurements of the Fermi cutoff of the W(110) crystal, yielding an average value of $83 \pm 3 \mathrm{meV}$ FWHM. (The error bars quoted here and throughout the paper represent a 95\%-confidence level, based on deviations from the mean of results obtained from fitting a large number of independent data sets.) Subtraction of the electron analyzer width yields a photon resolution of $73 \mathrm{meV}$, which is consistent with earlier determinations based on the much sharper Fermi edge of $\mathrm{Cu}$ at $90 \mathrm{~K}$. Analysis of both $\mathrm{Cu}$ and $\mathrm{W}$ Fermi edges confirms that the resolution function is very well approximated by a Gaussian.

The clean W(110) surface is ideally suited for studying the broadening mechanisms of surface-atom core levels. The $4 f$ states are long lived (i.e., the photopeaks are narrow) and there is no surface reconstruction. ${ }^{9}$ Moreover, for this close-packed surface, photoemission from subsurface layers should be indistinguishable from that from the bulk because the nearest- and next-nearestneighbor coordinations of the subsurface atoms are identical to the bulk values. A complete photoemission spectrum of the $\mathrm{W} 4 f$ lines from a freshly cleaned surface (see inset in Fig. 1) shows four lines. The $>2 \mathrm{eV}$ separation of the $j=\frac{5}{2}$ and $\frac{7}{2}$ spin-orbit doublet allows the individual components to be analyzed separately. Furthermore, the bulk and surface lines in the $4 f_{7 / 2}$ data are so well resolved and have such high statistics that independent values of the natural Lorentzian lifetime width, the Gaussian phonon and instrumental broadening, and the core-hole screening singularity index for each of the lines can be readily obtained.

The fit to the $4 f_{7 / 2}$ data is shown in Fig. 1. Its quality 


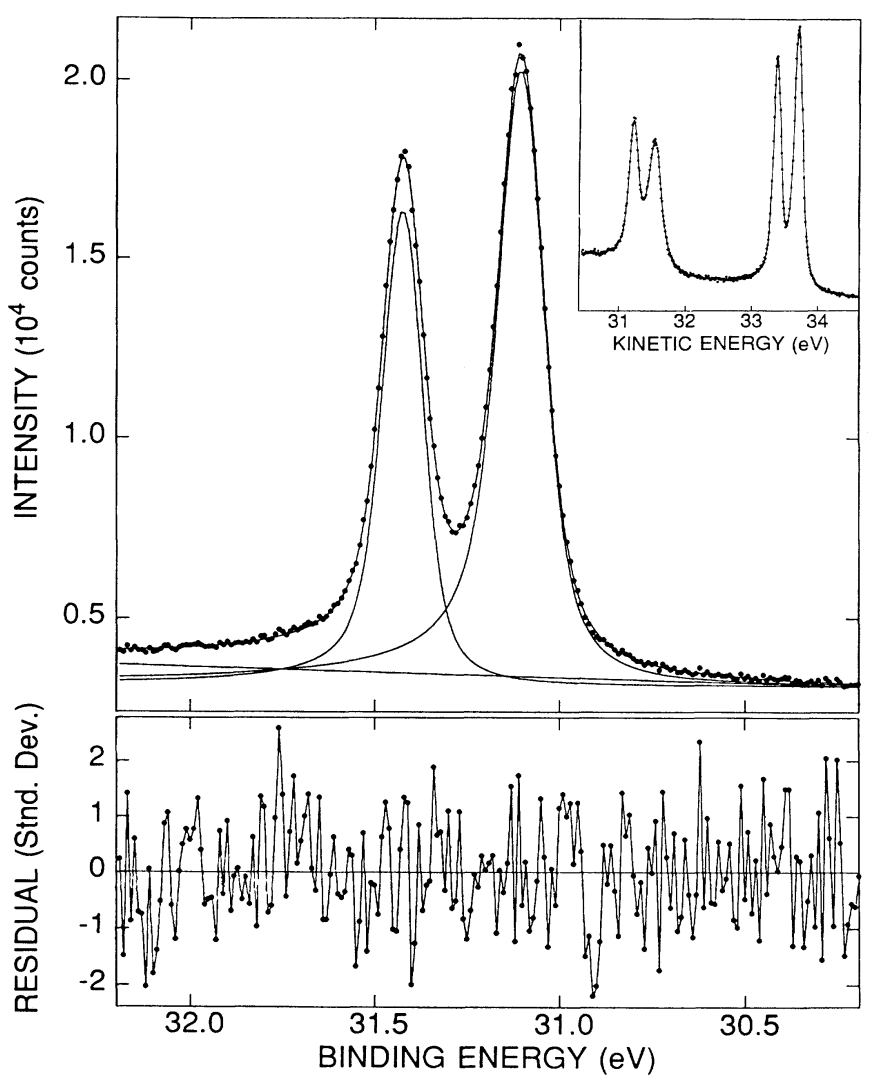

FIG. 1. Analysis of the $W f_{7 / 2}$ photoemission spectrum of a clean $\mathrm{W}(110)$ surface. The data, taken with $70-\mathrm{eV}$ photons, are fitted with a linear background and two fully independent lines, representing the surface (right) and bulk (left) contributions. The residuals of the fit are shown in the bottom panel. Inset: A scan encompassing the $\mathrm{W} 4 f_{5 / 2}$ and $4 f_{7 / 2}$ components.

can be critically assessed by the residuals, which exhibit no systematic deviations and show only the fluctuations due to counting statistics. This immediately demonstrates that one cannot hope to isolate a third component, viz., the signal from the second atomic layer, by least-squares analysis because its binding-energy shift is much smaller than the Gaussian width. Accordingly, we have restricted ourselves to a two-component analysis. Eighteen independent data sets were analyzed using two fully independent Doniach-Šnjic ${ }^{10}$ (DS) lines convolved with independent Gaussians to test the reproducibility of the output parameters. The resulting width and shape parameters for the bulk and surface lines contain a number of surprises.

(1) The surface-atom core-level shift is $321 \pm 1 \mathrm{meV}$. This value is decidedly larger than the splitting of 300 meV obtained from earlier data with lower resolution. ${ }^{11}$ In a related experiment we found that this splitting is reduced by adsorbed hydrogen [initially changing by about $-50 \mathrm{meV} / \mathrm{L}, 1 \mathrm{~L}$ (Langmuir) $=10^{-6}$ Torr sec, of $\mathrm{H}_{2}$ exposure], demonstrating that great care must be exercised

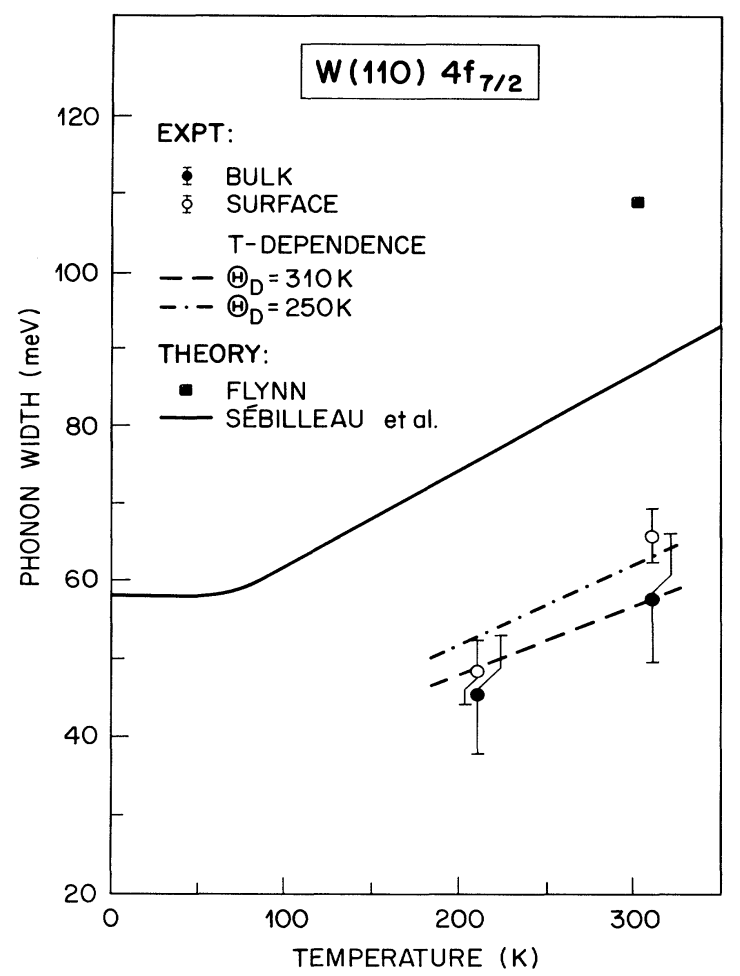

FIG. 2. Experimental phonon widths of the bulk and (110) surface W $4 f_{7 / 2}$ levels compared with the results of two theoretical calculations.

to maintain a clean surface during the measurement.

(2) We find that the independently determined Gaussian widths of the bulk and surface components are very similar at $210 \mathrm{~K}$ : $94.5 \pm 2.7$ and $96.0 \pm 1.4 \mathrm{meV}$, respectively. At $310 \mathrm{~K}$ the difference is only slightly larger, $100.8 \pm 4.4 \mathrm{meV}$ for the bulk and 105.7 \pm 1.4 meV for the surface. Subtracting the instrumental resolution in quadrature from the total Gaussian width results in an excess width for the bulk (surface) of 45.4 $\pm 7.6(48.4 \pm 5.5)$ at $210 \mathrm{~K}$ and $57.3 \pm 8.7(65.6 \pm 4.2)$ at $310 \mathrm{~K}$. The temperature dependence immediately identifies these widths, which are plotted in Fig. 2, as being due to phonons excited during the photoemission process. ${ }^{12}$ The room-temperature values are significantly smaller than the semiempirical estimate of $109 \mathrm{meV},{ }^{13}$ and both values are smaller than the theoretical estimates of 76 and $88 \mathrm{meV}^{14}$ for bulk $W$ at the corresponding temperatures (also shown in Fig. 2).

Although the measured phonon broadening is less than that expected from either calculation above, the results for the temperature dependence, along with the slight difference in surface and bulk values, are both consistent with an estimation based on the bulk and surface Debye temperatures of W(110). Using the bulk width at $310 \mathrm{~K}$ as the reference point and a bulk Debye temperature of $310 \mathrm{~K}^{15}$ gives the dashed line in Fig. 2. Keeping the coupling strength to the phonons constant but lowering 
the Debye temperature to $250 \mathrm{~K}$, a value appropriate for the W(110) surface, ${ }^{16}$ produces the dot-dashed curve which just intersects the error bars on the surface data. Thus, although the difference in phonon broadening is too small to discern any significant difference between the surface and the bulk, the data are consistent with a simple interpretation of a Debye-phonon excitation spectrum in the limit of a Gaussian line shape.

(3) In contrast to the small difference observed in the phonon broadening, the data clearly show that the corehole lifetime width is distinctly larger at the surface than in the bulk. Figure 3 demonstrates that there is no difficulty in separating the Gaussian and Lorentzian contri-

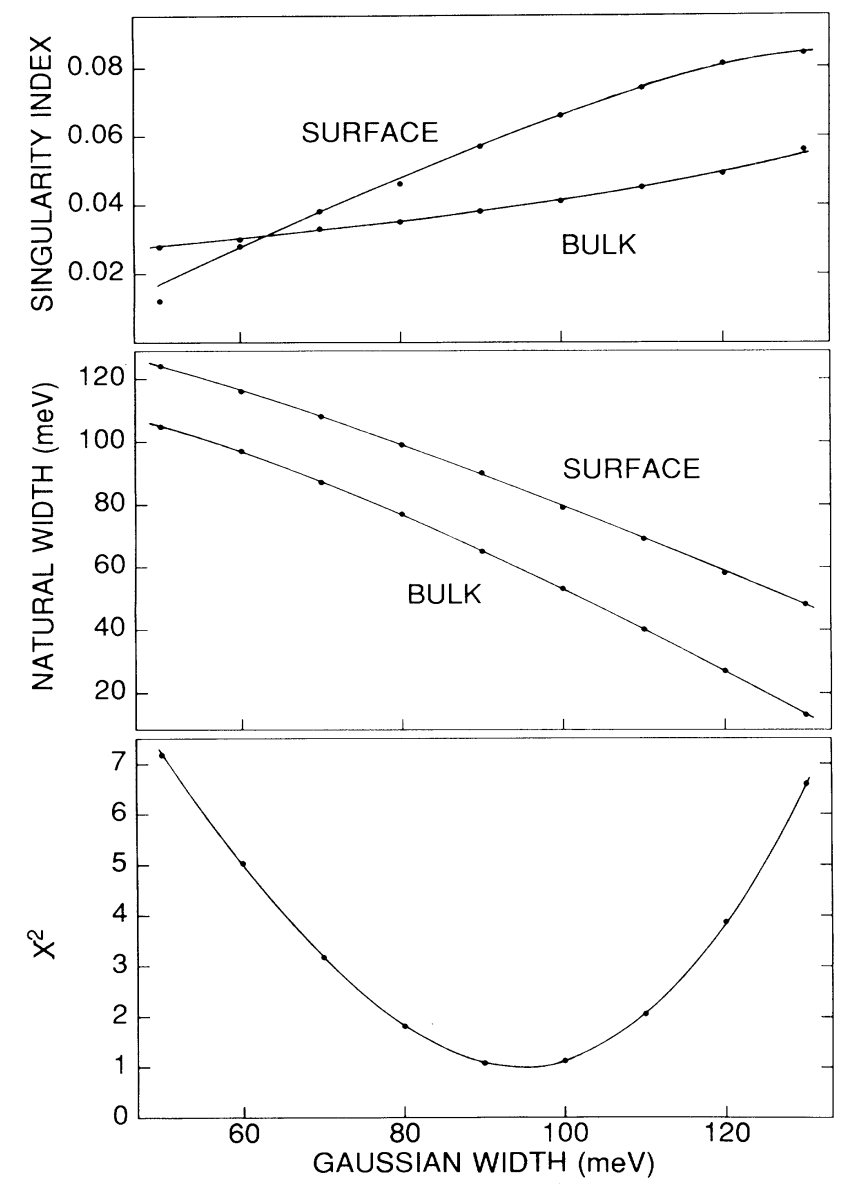

FIG. 3. Distinguishing between Gaussian and Lorentzian broadening. The bottom panel shows a cut through the $\chi^{2}$ surface of a least-squares analysis of the data in Fig. 1. For the purpose of this demonstration only, a common Gaussian width was used for both surface and bulk photopeaks, leaving the other parameters unconstrained. (The use of a common Gaussian width in these data is justified by the fact that the bulk and surface lines are so well separated.) The corresponding natural Lorentzian widths and singularity indices are shown above. The clearly defined minimum in $\chi^{2}$ demonstrates that the Lorentzian lifetime and Gaussian phonon broadenings are readily distinguishable in these data. butions to the linewidth. Analysis of the eighteen data sets yields a width of $60 \pm 3 \mathrm{meV}$ for the bulk and $84 \pm 3$ $\mathrm{meV}$ for the surface. The value for the lifetime width of the bulk line is in satisfactory agreement with earlier determinations, ${ }^{7}$ which were 55 and $61 \mathrm{meV}$.

Since the $4 f_{7 / 2}$ hole state decays almost entirely by $\mathrm{N}_{7} \mathrm{O}_{4,5} \mathrm{O}_{4,5}$ Auger transitions [the fluorescence yield is only $7 \times 10^{-6}$ (Ref. 17)], one can look for an explanation of the increased width at the surface by focusing on the properties of the metal $5 d$ band. Starting with the free W atom, there will be two opposing effects on the $4 f_{7 / 2}$ lifetime in forming the metal. On the one hand, the Auger decay rate will be enhanced because the $d$-band occupancy is increased both by reconfiguration in the initial state and by metallic $5 d$ core-hole screening in the final state. On the other hand, the Auger decay rate will be reduced by the delocalization of the $5 d$ conduction electrons, which decreases the overlap between the $4 f$ and $5 d$ orbitals. The larger lifetime width observed in the surface relative to the bulk, then, clearly implies that the reduced delocalization in the narrowed, more atomiclike surface $5 d$ band is the dominant mechanism.

(4) The singularity index, $\alpha$, of the bulk and surface lines are also different, $0.035 \pm 0.003$ and $0.063 \pm 0.003$, respectively (the rms deviations from the mean are small because the coupling of $\alpha$ to the other parameters is very weak). The small absolute values of $\alpha$, compared to, e.g., the simple metals $\mathrm{Na}, \mathrm{Li}, \mathrm{Mg}$, and $\mathrm{Al},{ }^{12}$ are a consequence of the larger fraction of screening charge residing in orbitals of higher angular momentum. The bulk value of $\alpha$ is close to the minimum value of $\frac{1}{32}$ obtainable for screening by $s, p, d$, and $f$ orbitals. By showing that the valence-band excitation spectrum is approximately constant from 0 to $5 \mathrm{eV}$, Sébilleau et al. ${ }^{14}$ have demonstrated that the DS line shape is appropriate for $W$. Their calculated values for $\alpha$ are, however, approximately the same for bulk and surface and are in agreement only with our surface value.

Our results for the $W(110)$ surface have significant implications on some of the earlier interpretations of surface-atom core-level measurements. ${ }^{1,5-8}$ For example, the present findings indicate that the excess width of the surface signal from $\mathrm{Ta}$ and other $\mathrm{W}$ surfaces is due almost solely to the increased natural width of the core hole, not the other broadening mechanisms which have been proposed. For the W(111) surface we have confirmed that the extant data are compatible with this conclusion, but the resolution of the data does not allow independent Gaussian- and Lorentzian-width parameters to be determined for the bulk and surface lines. In general, it should certainly not be assumed, as has been done in the past, that the natural width is the same in the bulk and surface, especially when it is determined primarily by Auger processes involving valence electrons. Moreover, the singularity index should not be assumed to be unchanged at the surface, even when the absolute bulk value is small. 
In summary, we have shown that the modification of the electronic properties at the surface readily accounts for the changes in the core-electron photoemission spectra from the first atomic layer of $\mathrm{W}(110)$. There is no anomalous phonon broadening, no evidence for crystalfield splitting, and no need to postulate unresolved lines from subsurface layers. The important phenomena at the surface are the changes in the natural width and the conduction-electron screening. These conclusions depart from all previous interpretations of surface-atom corelevel spectra and are not limited to W(110). We anticipate that the higher-energy resolution and beam intensities now obtainable from improved synchrotron sources and beam-line optics should open up a variety of systems from which new conclusions about surface photoemission will emerge.

We are indebted to Professor P. J. Estrup for the loan of the tungsten sample used in these experiments. The National Synchrotron Light Source at Brookhaven National Laboratory is supported by the U.S. Department of Energy, Division of Materials Sciences and Division of Chemical Sciences (DOE Contract No. DE-AC0276CH00016).

${ }^{1}$ For reviews, see P. H. Citrin and G. K. Wertheim, Phys. Rev. B 27, 3167 (1983); D. Spanjaard, C. Guillot, M. C. Desjonquères, G. Tréglia, and J. Lecante, Surf. Sci. Rep. 5, 1 (1985).

${ }^{2}$ P. H. Citrin, G. K. Wertheim, and Y. Baer, Phys. Rev. Lett. 41, 1425 (1978); Phys. Rev. B 27, 3160 (1983).
${ }^{3}$ P. J. Feibelman and D. R. Hamann, Solid State Commun. 31, 413 (1979); Phys. Rev. B 20, 1433 (1979).

${ }^{4}$ B. Johansson and N. Martensson, Phys. Rev. B 21, 4427 (1980); Helv. Phys. Acta 56, 405 (1983).

${ }^{5}$ J. F. van der Veen, F. J. Himpsel, and D. E. Eastman, Phys. Rev. Lett. 44, 189 (1980); Solid State Commun. 37, 555 (1981); 40, 57 (1981); Phys. Rev. B 25, 7388 (1982).

${ }^{6}$ G. Tréglia, M. C. Desjonquères, D. Spanjaard, Y. Lassailly, C. Guillot, Y. Jugnet, T. M. Duc, and J. Lecante, J. Phys. C 14, 3463 (1981); C. Guillot et al., J. Phys. C 15, 4023 (1982); 16, 1555 (1983); Solid State Commun. 50, 393 (1984); Phys. Rev. B 30, 5487 (1984).

${ }^{7}$ G. K. Wertheim, P. K. Citrin, and J. F. van der Veen, Phys. Rev. B 30, 4343 (1984); 38, 7820 (1988).

${ }^{8}$ K. G. Purcell, J. Jupille, G. P. Derby, and D. A. King, Phys. Rev. B 36, 1288 (1987); J. Jupille et al., Phys. Rev. B 39, 6871 (1989).

${ }^{9}$ M. A. van Hove and S. Y. Tong, Surf. Sci. 54, 91 (1976); J. W. Chung, S. C. Ying, and P. W. Estrup, Phys. Rev. Lett. 56, 749 (1986).

${ }^{10}$ S. Doniach and M. Šunjić, J. Phys. C 34, 285 (1970).

${ }^{11}$ T.-M. Duc, C. Guillot, Y. Lassailly, J. Lecante, Y. Jugnet, and J. C. Vedrine, Phys. Rev. Lett. 43, 789 (1979).

${ }^{12}$ P. H. Citrin, G. K. Wertheim, and Y. Baer, Phys. Rev. B 16, 4256 (1977).

${ }^{13}$ C. P. Flynn, Phys. Rev. Lett. 37, 1445 (1976).

${ }^{14}$ D. Sébilleau, G. Tréglia, M. C. Desjonquères, C. Guillot, D. Chauveau, and D. Spanjaard, J. Phys. C 20, 2647 (1987).

${ }^{15}$ N. W. Ashcroft and N. D. Mermin, Solid State Physics (Saunders, Philadelphia, 1976), p. 461.

${ }^{16}$ D. P. Jackson, Surf. Sci. 43, 431 (1974).

${ }^{17}$ E. J. McGuire, Phys. Rev. A 9, 1840 (1974). 